\title{
Recognition of Water-Acetonitrile-Water Cluster in a Tetraprotonated Picrate Salt of Octaaminocryptand
}

\author{
I. Ravikumar, P. S. Lakshminarayanan, E. Suresh ${ }^{*}$, Pradyut Ghosh* \\ Analytical Science Discipline, Central Salt \& Marine Chemicals Research Institute, \\ Bhavnagar 364002, INDIA, e-mail: pradyut@csmcri.org
}

1. Experimental Section $\quad$ S1

2. Table 1: Hydrogen-bonding parameter for Complexes $1 \quad$ S2

3. Table 2: Hydrogen-bonding parameter for Complexes $2 \quad$ S3

4. TG curve of $\mathbf{1}$ at heating rate of $10^{\circ} \mathrm{C} / \mathrm{min}$. S4

5. TG curve of 2 at heating rate of $10^{\circ} \mathrm{C} / \mathrm{min}$. S5

6. ORTEP diagram of $\mathbf{1}$ with atom numbering scheme $\quad \mathbf{S 6}$

7. ORTEP diagram of $\mathbf{2}$ with atom numbering scheme $\quad$ S7 


\section{Experimental Section}

Materials. Reagent-grade chemicals (Aldrich) were used as received. Picric acid and solvents were purchased from SD Fine Chemicals, India. All the solvents were purified prior to use.

Synthesis. The macrobicyclic cryptand $\mathbf{L}$ was synthesized according to previously published methods and a ${ }^{1} \mathrm{H}$ NMR spectrum of $\mathbf{L}$ was good agreement with previously reported data.

$\left[\mathrm{H}_{4} \mathbf{L}\left(\mathrm{CH}_{3} \mathrm{CN}\right)\left(\mathrm{H}_{2} \mathrm{O}\right)_{2}\right][\text { Picrate }]_{4} .2 .5 \mathrm{CH}_{3} \mathrm{CN}$, 1. Complex $\mathbf{1}$ was obtained by dissolving $\mathbf{L}$ (600 $\mathrm{mg}, 1 \mathrm{mmol})$ in acetonitrile $(15 \mathrm{ml})$ and adding $4 \mathrm{mmol}$ of picric acid in acetonitrile $(7 \mathrm{ml})$. The yellow solid formed was filtered and re-dissolved in hot acetonitrile $(20 \mathrm{ml})$. Crystals suitable for X-ray analysis were obtained by slow evaporation the solution at room temperature. Yield: 75\%. ${ }^{1} \mathrm{H}$ NMR (200 MHz, DMSO-d ${ }_{6}$ ): $\delta 2.63\left(\mathrm{t}, 12 \mathrm{H}, \mathrm{NCH}_{2}\right), 3.60\left(\mathrm{t}, 12 \mathrm{H}, \mathrm{NCH}_{2} \mathrm{CH}_{2}\right.$ ), $3.87\left(\mathrm{~s}, 12 \mathrm{H}, \mathrm{ArCH}_{2}\right), 7.1$ (s, 12H, ArH), 8.59 (s, 8H, PicH). ${ }^{13} \mathrm{C}$ NMR (50 MHz, DMSo-d 6 ): $\delta$ $48.57,53.99,54.85,128.01,129.337,130.51,139.068,143.11,156.21$.

$\left[\mathrm{H}_{3} \mathbf{L}\left(\mathrm{H}_{2} \mathrm{O}\right)_{1}\right][\text { Picrate }]_{3} .0 .5 \mathrm{H}_{2} \mathrm{O}$, 2. Complex 2 was obtained by dissolving $\mathbf{L}$ (600 mg, $1 \mathrm{mmol}$ ) in toluene $(15 \mathrm{ml})$ and adding $3 \mathrm{mmol}$ of picric acid in toluene $(7 \mathrm{ml})$. Crystals suitable for Xray analysis were obtained by slow evaporation of the solution at room temperature.

Yield: 78\%. ${ }^{1} \mathrm{H}$ NMR (200 MHz, DMSO-d 6 ): $\delta 2.62$ (t, 12H, $\mathrm{NCH}_{2}$ ), 3.27 (t, 12H, $\mathrm{NCH}_{2} \mathrm{CH}_{2}$ ), 3.946 (s, 12H, $\mathrm{ArCH}_{2}$ ), 7.19 (s, 12H, ArH), 8.59 (s, 6H, PicH). ${ }^{13} \mathrm{C}$ NMR (50 MHz, DMSO$\left.\mathrm{d}_{6}\right): \delta 48.33,53.25,55.64,126.33,130.861,132.12,138.57,143.599,154.327$. 


\section{Table 1: H-Bonding Table for 1}

\begin{tabular}{|c|c|c|c|}
\hline D-H•••A & $d(\mathrm{H} \bullet \bullet A)(\AA)$ & $\begin{array}{c}d(\mathrm{D} \bullet \bullet A) \\
(\AA)\end{array}$ & $<$ D-H...A $\left(^{\circ}\right)$ \\
\hline $\mathrm{N}(2)-\mathrm{H}(2 \mathrm{C}) \cdot \cdots \mathrm{O}(29)^{1}$ & $1.93(4)$ & $2.774(4)$ & $165(4)$ \\
\hline $\mathrm{N}(2)-\mathrm{H}(2 \mathrm{D}) \cdot \cdots \cdot \mathrm{O}(22)^{2}$ & $1.85(3)$ & $2.699(4)$ & $158(3)$ \\
\hline $\mathrm{N}(2)-\mathrm{H}(2 \mathrm{D}) \cdot \cdots \mathrm{O}(23)^{2}$ & $2.46(3)$ & $3.071(4)$ & $126(3)$ \\
\hline $\mathrm{N}(3)-\mathrm{H}(3 \mathrm{C}) \cdot \cdots \mathrm{O}(30)^{1}$ & 1.89 & $2.787(4)$ & 177 \\
\hline $\mathrm{N}(3)-\mathrm{H}(3 \mathrm{D}) \cdot \cdots \cdot \mathrm{O}(8)^{3}$ & 1.93 & $2.827(4)$ & 171 \\
\hline $\mathrm{N}(5)-\mathrm{H}(5 \mathrm{C}) \bullet \bullet \cdot \mathrm{O}(29)^{1}$ & 1.91 & $2.791(3)$ & 167 \\
\hline $\mathrm{N}(5)-\mathrm{H}(5 \mathrm{D}) \bullet \bullet \cdot \mathrm{O}(1)^{4}$ & 1.98 & $2.692(4)$ & 135 \\
\hline $\mathrm{N}(7)-\mathrm{H}(7) \cdot \bullet \cdot \mathrm{N}(21)^{1}$ & $2.30(4)$ & $3.173(5)$ & $175(3)$ \\
\hline $\mathrm{N}(8)-\mathrm{H}(8 \mathrm{C}) \cdot \cdots \mathrm{O}(15)^{1}$ & $1.94(4)$ & $2.789(4)$ & $161(4)$ \\
\hline $\mathrm{N}(8)-\mathrm{H}(8 \mathrm{D}) \cdot \cdots \mathrm{O}(30)^{1}$ & $1.92(3)$ & $2.832(4)$ & $177(4)$ \\
\hline $\mathrm{O}(29)-\mathrm{H}(29 \mathrm{D}) \cdot \cdots \mathrm{N}(7)^{1}$ & $1.76(5)$ & $2.729(4)$ & $173(4)$ \\
\hline $\mathrm{O}(29)-\mathrm{H}(29 \mathrm{E}) \cdot \cdots \cdot \mathrm{N}(22)^{1}$ & $2.10(4)$ & $2.863(4)$ & $152(3)$ \\
\hline $\mathrm{O}(30)-\mathrm{H}(30 \mathrm{D}) \cdot \cdots \cdot \mathrm{N}(22)^{1}$ & $2.14(5)$ & $2.875(4)$ & $139(4)$ \\
\hline $\mathrm{O}(30)-\mathrm{H}(30 \mathrm{E}) \cdots \mathrm{N}(6)^{1}$ & $2.10(5)$ & $2.782(5)$ & $170(5)$ \\
\hline $\mathrm{C}(1)-\mathrm{H}(1 \mathrm{~A}) \cdots \mathrm{O}(19)^{5}$ & 2.48 & $3.315(4)$ & 144 \\
\hline $\mathrm{C}(3)-\mathrm{H}(3 \mathrm{~B}) \cdot \cdots \cdot \mathrm{O}(16)^{3}$ & 2.54 & $3.316(4)$ & 137 \\
\hline $\mathrm{C}(6)-\mathrm{H}(6) \cdots \cdot \mathrm{O}(10)^{2}$ & 2.53 & $3.211(4)$ & 130 \\
\hline $\mathrm{C}(11)-\mathrm{H}(11 \mathrm{~A}) \cdot \cdots \cdot \mathrm{O}(14)^{3}$ & 2.39 & $2.934(5)$ & 115 \\
\hline $\mathrm{C}(14)-\mathrm{H}(14 \mathrm{~B}) \cdots \mathrm{O}(23)^{2}$ & 2.56 & $3.343(4)$ & 138 \\
\hline $\mathrm{C}(15)-\mathrm{H}(15 \mathrm{~A}) \cdots \mathrm{O}(23)^{2}$ & 2.58 & $3.315(4)$ & 133 \\
\hline $\mathrm{C}(27)-\mathrm{H}(27 \mathrm{~B}) \cdot \cdots \mathrm{N}(14)^{1}$ & 2.59 & $3.516(5)$ & 161 \\
\hline $\mathrm{C}(32)-\mathrm{H}(32) \cdots \cdot \mathrm{O}(9)^{3}$ & 2.56 & $3.272(4)$ & 133 \\
\hline $\mathrm{C}(34)-\mathrm{H}(34 \mathrm{~A}) \cdot \cdots \mathrm{O}(16)^{1}$ & 2.59 & $3.187(4)$ & 120 \\
\hline $\mathrm{C}(34)-\mathrm{H}(34 \mathrm{~B}) \cdot \cdots \mathrm{O}(9)^{3}$ & 2.40 & $3.298(4)$ & 153 \\
\hline $\mathrm{C}(35)-\mathrm{H}(35 \mathrm{~A}) \cdots \cdot \mathrm{O}(16)^{1}$ & 2.59 & $3.172(4)$ & 119 \\
\hline
\end{tabular}




$\begin{array}{cccc}\mathrm{C}(35)-\mathrm{H}(35 \mathrm{~B}) \cdots \cdot \mathrm{O}(8)^{3} & 2.53 & 3.472(4) & 164 \\ \mathrm{C}(36)-\mathrm{H}(36 \mathrm{~A}) . . \mathrm{O}(27)^{3} & 2.49 & 3.320(4) & 143 \\ \mathrm{C}(63)-\mathrm{H}(63 \mathrm{~B}) \cdots \cdot \mathrm{O}(11)^{7} & 2.46 & 3.238(5) & 138 \\ \mathrm{C}(65)-\mathrm{H}(65 \mathrm{~B}) \cdots \cdot \mathrm{O}(1)^{8} & 2.52 & 3.474(5) & 178 \\ \mathrm{C}(65)-\mathrm{H}(65 \mathrm{C}) \cdots \cdot \mathrm{O}(13)^{9} & 2.59 & 3.466(5) & 151\end{array}$

1. x,y,z 2. x,1+y,z 3. 1/2-x,1/2+y,1/2-z 4. 1/2-x,-1/2+y,1/2-z 5. -1/2+x,1/2+y,z 6. -x,1-y,-z 7. 1/2$\mathrm{x}, 1 / 2-\mathrm{y},-\mathrm{z} 8 . \mathrm{x}, 2-\mathrm{y},-1 / 2+\mathrm{z} 9.1 / 2+\mathrm{x}, 1 / 2+\mathrm{y}, \mathrm{z}$ 


\section{Table 2: H-Bonding Table for 2}

\begin{tabular}{|c|c|c|c|}
\hline D-H•••A & $d(\mathrm{H} \bullet \bullet A)(\AA)$ & $\begin{array}{c}d(\mathrm{D} \bullet \bullet A) \\
(\AA)\end{array}$ & $<$ DHA $\left(^{\circ}\right)$ \\
\hline $\mathrm{N}(2)-\mathrm{H}(2 \mathrm{C}) \bullet \bullet-\mathrm{O}(22)^{1}$ & 1.85 & $2.597(4)$ & 171 \\
\hline $\mathrm{N}(4)-\mathrm{H}(4 \mathrm{C}) \bullet \bullet \cdot \mathrm{N}(3)^{1}$ & $2.35(5)$ & $2.921(4)$ & $121(4)$ \\
\hline $\mathrm{N}(4)-\mathrm{H}(4 \mathrm{C}) \bullet \bullet \mathrm{N}(8)^{1}$ & $2.43(5)$ & $2.902(4)$ & 113(4) \\
\hline $\mathrm{N}(5)-\mathrm{H}(5 \mathrm{C}) \bullet \bullet \cdot \mathrm{O}(8)^{2}$ & 1.99 & $2.856(4)$ & 160 \\
\hline $\mathrm{N}(5)-\mathrm{H}(5 \mathrm{C}) \bullet \bullet \mathrm{O}(9)^{2}$ & 2.39 & $2.936(4)$ & 119 \\
\hline $\mathrm{N}(5)-\mathrm{H}(5 \mathrm{D}) \bullet \bullet \cdot \mathrm{O}(22)^{1}$ & 1.71 & $2.578(4)$ & 162 \\
\hline $\mathrm{N}(7)-\mathrm{H}(7 \mathrm{C}) \bullet \bullet \cdot \mathrm{O}(22)^{1}$ & $1.85(5)$ & $2.641(4)$ & 171(3) \\
\hline $\mathrm{N}(7)-\mathrm{H}(7 \mathrm{D}) \bullet \bullet \mathrm{O}(15)^{3}$ & $1.93(4)$ & $2.834(5)$ & $157(3)$ \\
\hline $\mathrm{N}(7)-\mathrm{H}(7 \mathrm{D}) \bullet \bullet \mathrm{O}(16)^{3}$ & $2.25(3)$ & $2.818(4)$ & $123(3)$ \\
\hline $\mathrm{O} 22-\mathrm{H}(22 \mathrm{C}) \cdots \mathrm{N}(7)^{1}$ & 1.95 & $2.641(4)$ & 148 \\
\hline $\mathrm{C}(3)-\mathrm{H}(3 \mathrm{~A}) \bullet \bullet \cdot \mathrm{O}(7)^{4}$ & 2.49 & $3.115(4)$ & 122 \\
\hline $\mathrm{C}(3)-\mathrm{H}(3 \mathrm{~B}) \cdot \bullet \cdot \mathrm{O}(21)^{3}$ & 2.33 & $3.269(4)$ & 161 \\
\hline $\mathrm{C}(8)-\mathrm{H}(8) \cdot \bullet \cdot \mathrm{O}(4)^{5}$ & 2.57 & $3.191(4)$ & 124 \\
\hline $\mathrm{C}(12)-\mathrm{H}(12 \mathrm{~A}) \cdot \bullet \cdot \mathrm{O}(10)^{6}$ & 2.38 & $3.256(4)$ & 149 \\
\hline $\mathrm{C}(14)-\mathrm{H}(14 \mathrm{~B}) \cdot \bullet \mathrm{O}(1)^{4}$ & 2.34 & $3.292(5)$ & 168 \\
\hline $\mathrm{C}(23)-\mathrm{H}(23 \mathrm{~B}) \bullet \cdot \bullet \mathrm{O}(17)^{5}$ & 2.44 & $3.357(4)$ & 158 \\
\hline $\mathrm{C}(26)-\mathrm{H}(26 \mathrm{~B}) \cdot \bullet \cdot \mathrm{O}(13)^{2}$ & 2.51 & $3.204(6)$ & 129 \\
\hline $\mathrm{C}(32)-\mathrm{H}(32) \cdots \cdot \mathrm{O}(19)^{7}$ & 2.48 & $3.380(5)$ & 164 \\
\hline $\mathrm{C}(36)-\mathrm{H}(36 \mathrm{~B}) \cdot \bullet \cdot \mathrm{O}(6)^{8}$ & 2.50 & $3.235(5)$ & 132 \\
\hline
\end{tabular}




\section{TG curve of 1}

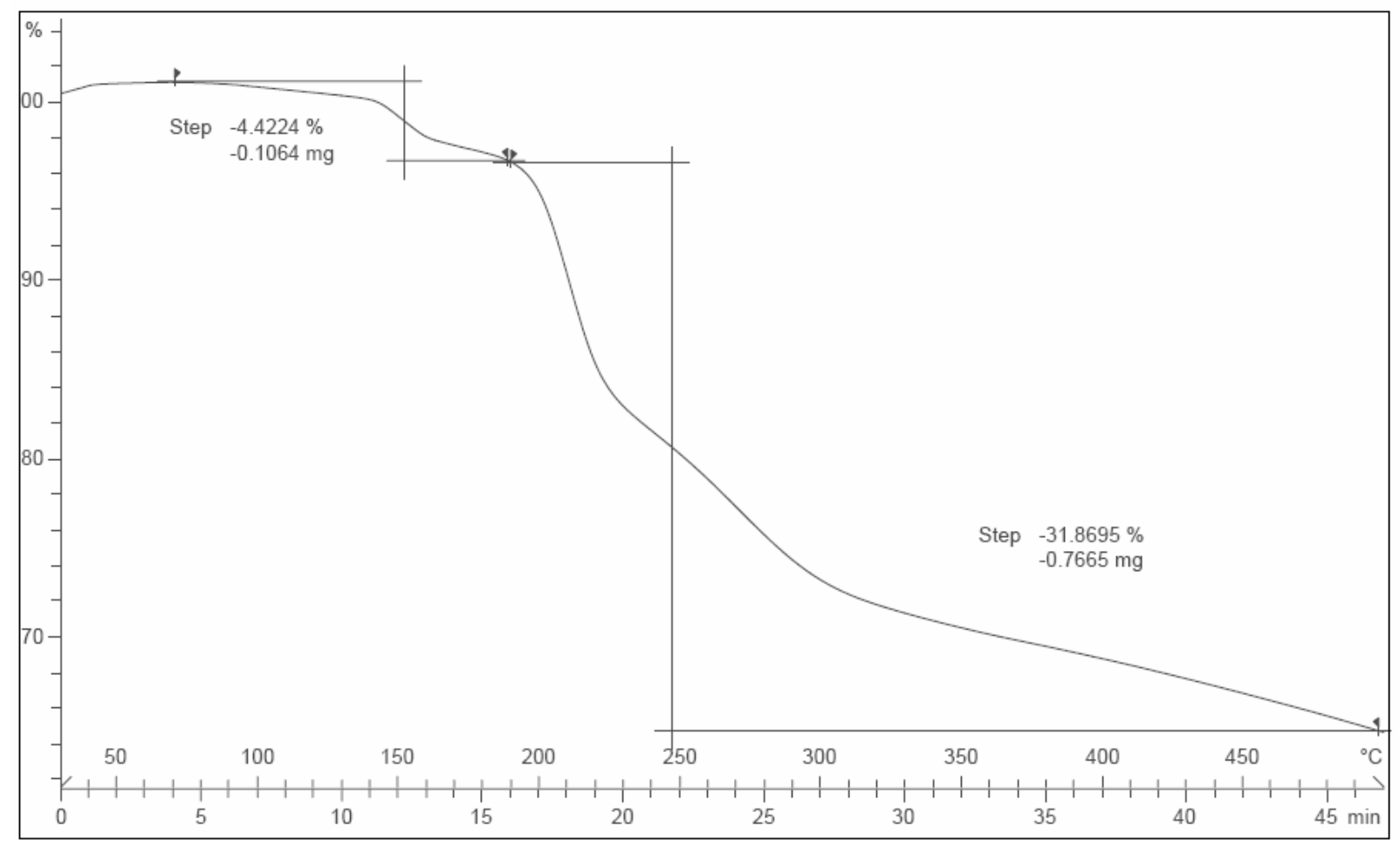

S4 


\section{TG curve of 2}

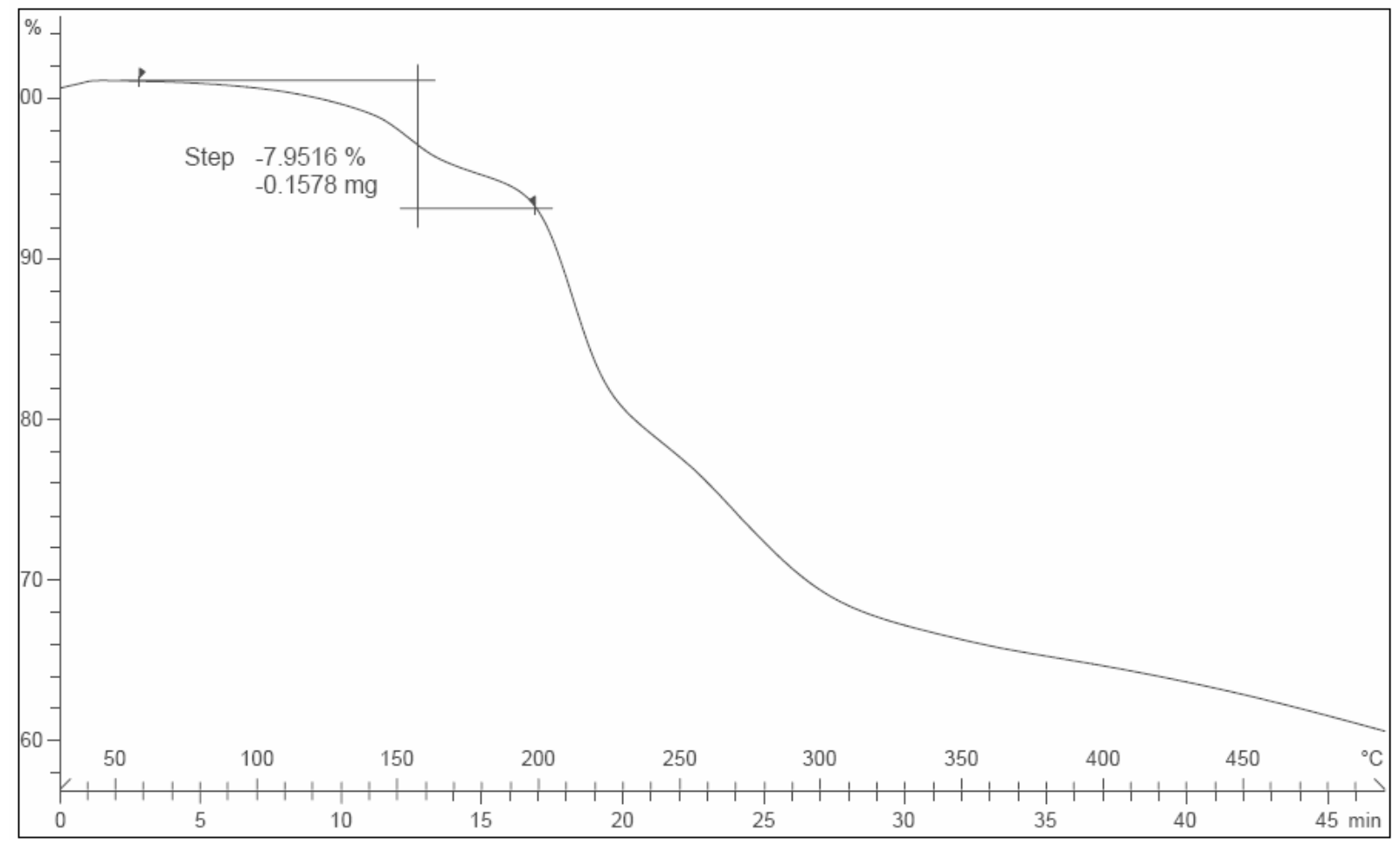

S5 


\section{ORTEP DIAGRAM OF 1}

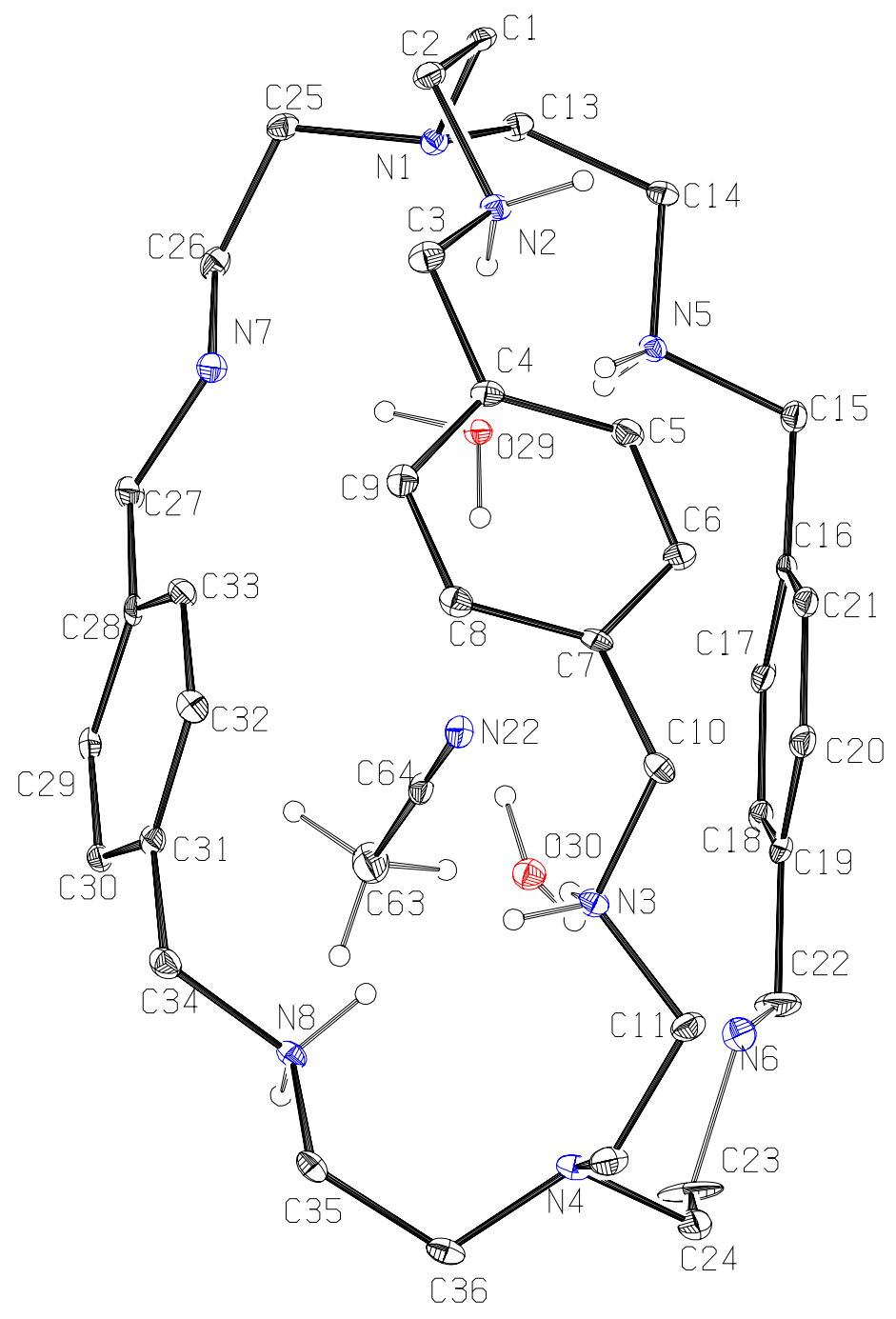




\section{ORTEP DIAGRAM OF 2}

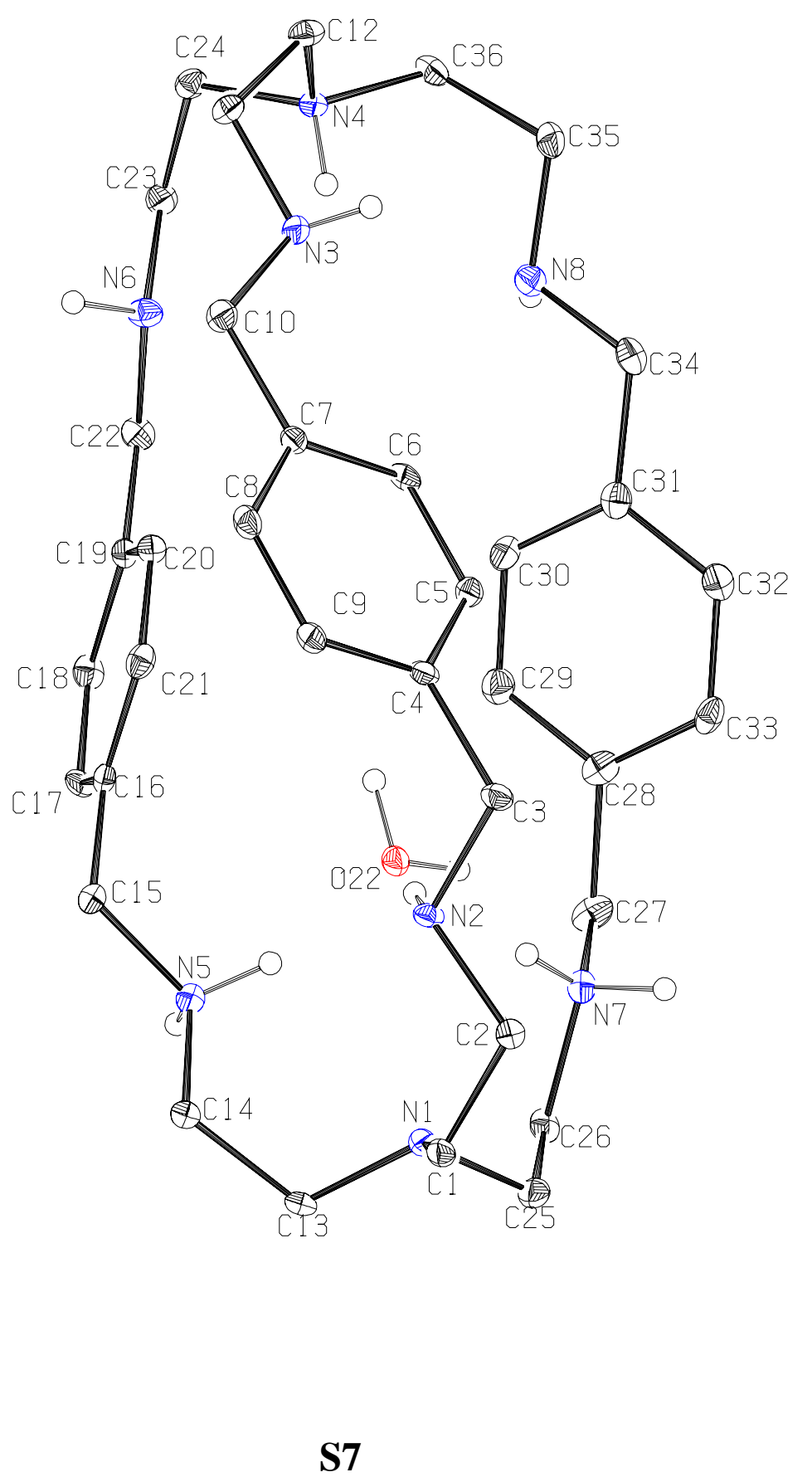

